\title{
The science case for the Next Generation AO system at W. M. Keck Observatory
}

C. Max, E. McGrath, D. Gavel, D. Le Mignant, P. Wizinowich, et al.

C. Max, E. McGrath, D. Gavel, D. Le Mignant, P. Wizinowich, R. Dekany, "The science case for the Next Generation AO system at W. M. Keck Observatory," Proc. SPIE 7015, Adaptive Optics Systems, 701507 (10 July 2008); doi: 10.1117/12.790173

EDent: SPIE Astronomical Telescopes + Instrumentation, 2008, Marseille, France 


\title{
The Science Case for the Next Generation AO System at W. M. Keck Observatory
}

\author{
C. Max ${ }^{* a}$, E. McGrath ${ }^{\mathrm{a}}$, D. Gavel ${ }^{\mathrm{a}}$, D. Le Mignant ${ }^{\mathrm{b}}$, P. Wizinowich ${ }^{\mathrm{b}}$, R. Dekany ${ }^{\mathrm{c}}$ \\ ${ }^{a}$ University of California at Santa Cruz, 1156 High Street, Santa Cruz, CA 95064; \\ ${ }^{\mathrm{b}}$ W. M. Keck Observatory, 65-1120 Mamalahoa Hwy, Kamuela, HI 96743 \\ ${ }^{\mathrm{C}}$ Caltech Optical Observatories, California Institute of Technology
}

\begin{abstract}
The W. M. Keck Observatory is designing a new adaptive optics system providing precision AO correction in the near infrared, good correction at visible wavelengths, and multiplexed spatially resolved spectroscopy. We discuss science cases for this Next Generation AO (NGAO), and show how the system requirements were derived from these science cases. Key science drivers include asteroid companions, planets around low-mass stars, general relativistic effects around the Galactic Center black hole, nearby active galactic nuclei, and high-redshift galaxies (including galaxies lensed by intervening galaxies or clusters). The multi-object AO-corrected integral field spectrograph will be optimized for high-redshift galaxy science.
\end{abstract}

Keywords: adaptive optics, multi-object adaptive optics, MOAO

\section{INTRODUCTION}

The Next Generation Adaptive Optics (NGAO) system at W. M. Keck Observatory will have three key goals: To provide diffraction-limited performance in near-infrared (K-band Strehl ratio $>80 \%$ ); to provide good AO correction at red wavelengths $(0.7-1.0 \mu \mathrm{m})$; and to provide increased sky coverage and multiplexing. The architecture chosen to achieve these goals is what we call a cascaded relay. Light coming from the telescope first encounters a wide-field adaptive optics relay, in order to feed pickoffs for AO-corrected infrared tip-tilt sensors, a near-infrared multiplexed deployable integral field spectrograph (IFU), and multiple laser guide star wavefront sensors. After passing through this wide-field relay, light encounters a high-Strehl narrow-field AO relay which feeds on-axis instruments such as imagers and a single high-performance IFU. Descriptions of the adaptive optics system and its components may be found in the following papers in this volume: 7015-36 (Wizinowich), 7015-76 (Dekany), 7015-93 (Velur), 7015-128 (Dekany), 7015225 (Le Mignant), 7015-226 (Gavel), 7015-239 (Sivaramakrishnan).

Here we describe the main science drivers, give an overview of the process for determining the science requirements, and highlight some key results.

\subsection{Key Science Drivers and Science Drivers}

The Science Team of the Keck NGAO project is charged with three tasks: 1) identifying compelling science cases that will set the requirements for NGAO performance, 2) deriving from these the science requirements for the NGAO system, and 3) when design trade-offs must be made, ensuring that the NGAO system will be built with capabilities that enable key science cases to be carried out to the greatest extent possible.

To prepare the science case for Keck NGAO, we analyzed two classes of science cases: "Key Science Drivers" and "Science Drivers. "Key Science Drivers" were those science cases that place the strongest or most technologically challenging demands on the performance of the NGAO system and its science instruments. These are the science cases that we have used to drive the performance requirements for the AO system and instruments. "Science Drivers" (not "Key") were included to assure that the NGAO system is sufficiently flexible to deal with the broad range of science that users will demand over the lifetime of the NGAO system. Typically, "Science Drivers" do not strongly push the state of the art of the AO system itself; rather they require specific types of coordination between the AO system, the instruments, and the telescope, or they help define parameters such as the full wavelength range or the required field of

*max@ucolick.org; phone 831-459-2049

Adaptive Optics Systems, edited by Norbert Hubin, Claire E. Max, Peter L. Wizinowich, Proc. of SPIE Vol. 7015, 701507, (2008)

0277-786X/08/\$18 $\cdot$ doi: $10.1117 / 12.790173$

Proc. of SPIE Vol. $7015701507-1$ 
view of the instruments. We have defined and analyzed five "Key Science Drivers" and nine "Science Drivers." These were selected because they represented important astrophysics within the Keck user community that would clarify the requirements on the NGAO system from a variety of perspectives.

The "Key Science Drivers" that we analyzed are as follows, in inverse order of distance from Earth: 1) Galaxy Assembly and Star Formation History, 2) Nearby Active Galactic Nuclei, 3) Precision Astrometry: Measurement of General Relativistic Effects at the Galactic Center, 4) Imaging and Characterization of Extrasolar Planets around Nearby Stars, and 5) Multiplicity of Minor Planets in our Solar System

The additional "Science Drivers" that we analyzed are as follows: Quasar Host Galaxies, Strong Gravitational Lensing, Astrometry Science in Sparse Fields, Resolved Stellar Populations in Crowded Fields, Debris Disks and Young Stellar Objects, Size, Shape, and Composition of Minor Planets, Characteristics of Gas Giant Planets, their Satellites, and Rings, Characteristics of Ice Giant Planets and their Rings, and Backup Science (to assure that the system will enable the appropriate observing modes).

\section{ANALYSIS OF KEY SCIENCE DRIVERS}

In this section we give a brief overview of each Key Science Driver. A more detailed treatment is found in the NGAO Science Case Requirements Document $\left[^{1}\right]$.

\subsection{Galaxy Assembly and Star Formation History}

At redshifts $\mathrm{z}>1$, galaxies have shrunk to angular sizes of approximately 1 arc sec, so that seeing based observations are not useful at uncovering morphologies and internal kinematics. At the epoch of greatest star formation and AGN activity around $\mathrm{z} \sim 2.5$, traditional optical lines of $\mathrm{H \alpha}$, OIII and OII are nicely shifted into the $\mathrm{K}, \mathrm{H}$ and $\mathrm{J}$ bands respectively. Integral field spectrographs such as OSIRIS at Keck $\left[{ }^{2}\right]$ and SINFONI at the VLT $\left[{ }^{3}\right]$ are now starting to dissect some of the brightest galaxies at this epoch using the increased sky coverage provided by laser guide stars. With the sensitivity gain and increased sky coverage possible with Keck NGAO, a wealth of new science topics can be addressed. These include the relationship between AGN and their host galaxies: radio galaxies and quasars have very strong emission lines and complex kinematics. In "normal" galaxies, the redshift range from 1.5 to 2.5 is the key era for the birth of their first stars and the formation of the major architectural components of the galaxy, the bulge and disk. Measuring the morphology of star formation, the kinematics of proto-disks, the internal velocity dispersions and metallicity gradients (e.g. from the NII/Ho ratio) will allow us to witness the birth of galaxies like the Milky Way.

The study of high-redshift galaxies is a powerful driver for multiplexed observations, for example using deployable integral field unit (IFU) spectrographs (imaging spectrographs that record a spectrum at every pixel in an image). With current-generation instruments, it is extremely challenging to observe a representative sample of sources due to the uncertainties inherent in long-slit spectroscopy (e.g. slit misalignment with kinematic axes), seeing-limited integral field spectroscopy (e.g. loss of information on scales smaller than the seeing disk), or a single-object IFU with currentgeneration adaptive optics (for which integration times are prohibitively long for obtaining a large statistical sample). Given the areal densities of 1 to 10 targets per square arc minute on the sky depending on the target selection criteria, a deployable integral field spectrograph (IFU) used with multi-conjugate or multi-object adaptive optics (MCAO or MOAO) would be capable of simultaneously observing many targets within a several square arc minute field, permitting the compilation of a large representative sample with a minimum of observing time. A high-Strehl NGAO system with multi-object IFU capability and low background would represent a major advance towards reliable kinematic and chemical data for a large sample of high redshift galaxies.

Based on the numerical simulations of Law et al. $\left[{ }^{4}\right]$ and the observed performance of the OSIRIS spectrograph at Keck, Figure 1 shows the significant improvement in signal to noise ratio predicted for the NGAO system relative to today's Keck 2 laser guide star AO. With the assumptions shown in the figure caption, NGAO would yield a signal to noise ratio 3 - 6 times higher than with the current Keck LGS AO system for the same exposure times, or would allow observations with the same signal to noise ratio to be achieved in much shorter integration times. Adding multiple IFUs would increase the scientific throughput still more. For example with 6 deployable integral field units, one would see gains of factors of 50 or more in the completion rate for survey-level programs, relative to the current LGS AO OSIRIS system. This is an astounding advance in the potential of AO systems to complete deep spectroscopic surveys of the distant universe. 


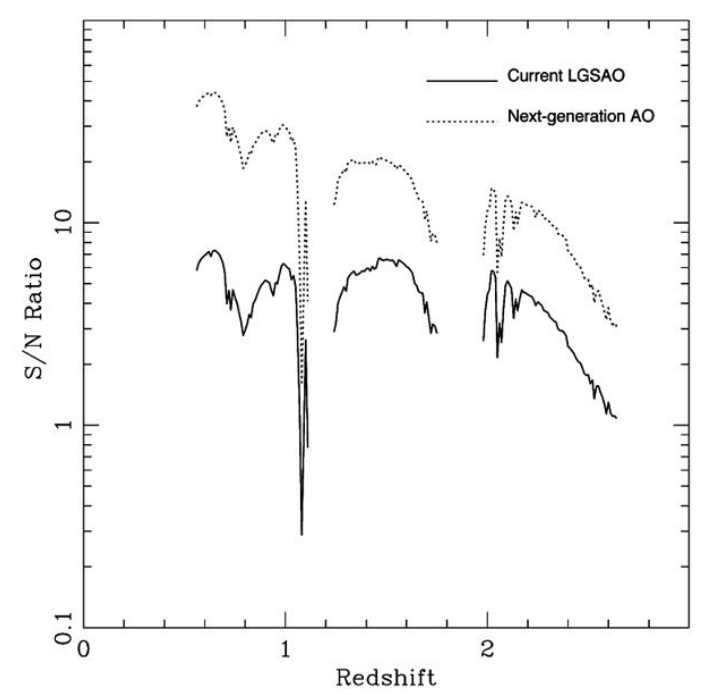

Figure 1 Predicted signal to noise ratios for an OSIRIS-like integral field spectrograph observing the $\mathrm{H} \alpha$ emission from galaxies at various redshifts. Signal-to-noise ratio for NGAO (upper curve) is compared with that for today's OSIRIS with LGS AO on Keck 2 (bottom curve) in a calculation by David Law. Assuming that the distant galaxy has at least some point-like substructure, for redshifts $0.6<\mathrm{z}<2.3 \mathrm{NGAO}$ shows a factor of 3 to 6 improvement in signal to noise ratio. Here we have used IFU lenslet sizes of 0.1 arc sec, and required that the added background due to the NGAO system be less than $30 \%$ of the unattenuated background due to the telescope plus sky.

A key conclusion from this analysis is the following: The total background seen at the focal plane of all spectrographs being fed by NGAO shall be less than $130 \%$ of the unattenuated sky plus telescope background (at $2209 \mathrm{~nm}$ and at a spectral resolution $\lambda / \Delta \lambda=5000$ ), with a goal of less than $120 \%$. The implications of this requirement are that the NGAO system should be cooled to about $260 \mathrm{~K}$. Other requirements from this key science driver include the following: the spectral resolution should be greater than $\mathrm{R} \sim 3000$ in order to effectively resolve out $\mathrm{OH}$ sky features and distinguish $\mathrm{H \alpha}$ from [N II] emission, and the sky coverage fraction should be at least $30 \%$ with encircled energy radius $<70$ mas.

\subsection{Nearby Active Galactic Nuclei}

It has become increasingly clear that black holes play a key role in galaxy formation and evolution. The most important evidence for a close connection between black hole growth and galaxy evolution comes from the observed correlations between black hole mass and the bulge velocity dispersion of the host galaxy (the "M- $\sigma$ relation"), and from the correlation between black hole mass and bulge mass $\left[^{5}\right]$. Despite the fact that black holes contain only about $0.1 \%$ of the mass of their host bulge, their growth is evidently constrained very tightly by the kiloparsec-scale properties of their environment. In addition, simulations and theory have highlighted the importance of feedback from active galactic nuclei (AGNs), in the form of winds or outflows which can serve to shut off AGN fueling and potentially expel a significant fraction of the host galaxy's gas into the intergalactic medium following a major merger. AGN feedback is frequently invoked as a mechanism to limit black hole growth and to shut off star formation in early-type galaxies, but observational evidence for this scenario remains sketchy.

Key observational goals in this field include the accurate determination of the demographics of black holes in nearby galaxies over the widest possible range in black hole mass, investigations of the redshift evolution of the M- $\sigma$ relation, and studies of the host galaxies of AGNs out to high redshifts to determine bulge luminosities, stellar populations, and emission-line kinematics. AO observations will be crucial in addressing these issues over the next decade.

Detections of the black holes in the Milky Way and in the megamaser galaxy NGC 4258 remain the "gold standard" in this field, but the majority of black hole detections to date have been done with the Hubble Space Telescope (HST) and are limited to galaxies without significant dust obscuration. In the best cases, observations of spatially resolved stellar or gas kinematics can yield black hole masses with uncertainties in the range $\sim 10-20 \%$, which is sufficient for an accurate determination of the M- $\sigma$ relation. Currently, although there are about 30 kinematics-based detections of massive black holes, the slope and the amount of scatter in the M- $\sigma$ relation remain somewhat controversial. In particular, the extreme ends of the black hole mass spectrum, above $10^{9}$ and below $10^{7}$ solar masses, remain poorly determined. Improvements in angular resolution lead directly to increased accuracy in black hole mass measurements; NGAO at Keck can therefore be the next significant new capability in this field. 
For black hole detection, NGAO offers two important advantages over current capabilities. First: in the near-IR, compared with current LGS AO the improved PSF quality and stability will significantly reduce the measurement uncertainty in black hole masses. Second: an AO capability in the I band will open up the possibility of using the Ca II triplet lines, giving a PSF core that is narrower than in the near-IR, and which will extend the distance out to which the

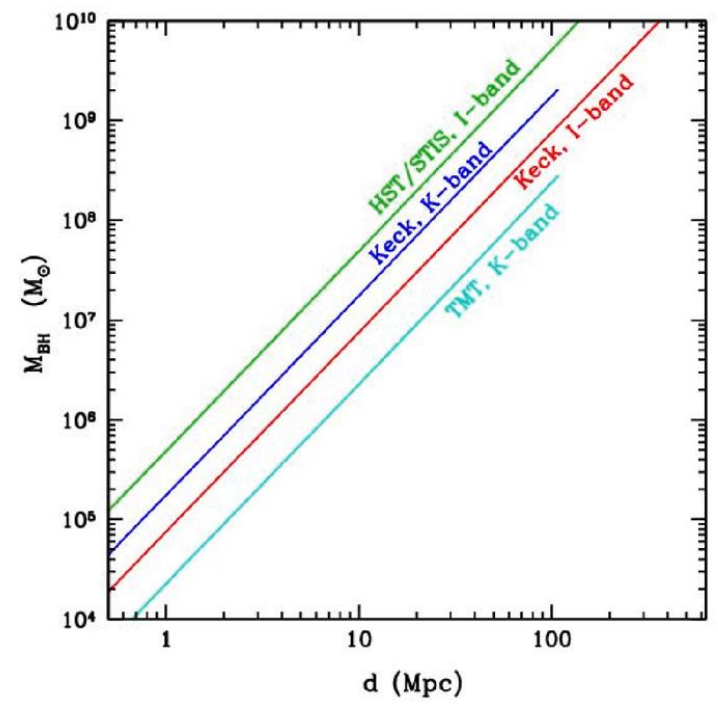

Figure 2 Minimum detectable black hole mass as a function of galaxy distance. We assume that black holes follow the local M$\sigma$ relation, and assume that a minimum of two resolution elements across the black hole's radius of influence is needed. For Keck $\mathrm{NGAO}$, this figure assumes a PSF core with FWHM $=0.053$ " in $\mathrm{K}$, and 0.035 " in I band. Minimum detectable black hole mass scales approximately as (distance $\mathrm{x}$ angular resolution) $)^{2}$. For distances beyond $\sim 100 \mathrm{Mpc}$, the $\mathrm{CO}$ bandhead is redshifted out of the K-band and is no longer observable; hence I-band spectroscopy using the Ca II triplet lines is needed. The line shown for the Thirty Meter Telescope (TMT) assumes a diffraction-limited PSF core in the K-band. Based on calculations by Aaron Barth.

most massive black holes can be detected. The minimum black hole mass detectable with a given angular resolution can be roughly estimated under the assumption that black holes lie on the M- $\sigma$ relation. As Figure 2 shows, Keck NGAO in the K-band would offer better sensitivity to black holes than that of HST/STIS in I band. In comparison with NGAO at K-band, for a given limiting distance an I-band NGAO capability with a PSF core FWHM of $\leq 0.035$ " can allow detections of black holes smaller in mass by approximately a factor of two.

Requirements based on this key science driver include the following: observing wavelength range $0.85-2.4 \mu \mathrm{m}$ including I-band capability with a PSF core FWHM of $\leq 0.035$ ". This science case is one of the drivers for doing diffraction limited spectroscopy at wavelengths (e.g. $850 \mathrm{~nm}$ ) shorter than 1 micron.

\subsection{Precision Astrometry: Measurements of General Relativity Effects in the Galactic Center}

The proximity of our Galaxy's center presents a unique opportunity to study a massive black hole and its environs at much higher spatial resolution than can be brought to bear on any other galaxy. In the last decade, near-IR observations with astrometric precisions of $<1$ mas and radial velocity precision of $20 \mathrm{~km} / \mathrm{s}$ have enabled the measurement of orbital motions for several stars near the Galactic Center, revealing a central dark mass of about $4 \times 10^{6} \mathrm{M}_{\text {Sun }}\left[{ }^{6} 78{ }^{9}\right.$ ]. Radio VLBA observations have now resolved the central compact object to within several multiples of the event horizon, indicating that the central mass is confined to a radius smaller than $1 \mathrm{AU}\left[{ }^{10}\right]$. These observations provide the most definitive evidence yet for the existence of massive black holes in the centers of galaxies. The orbital motions now also provide the most accurate measurement of the Galactic Center distance $\mathrm{R}_{0}$, constraining it to within a few percent [ $\left.{ }^{11}\right]$.

Due to the crowded stellar environment at the Galactic Center and the strong line-of-sight optical absorption, tracking the stellar orbits requires the high angular resolution, near-IR imaging capabilities of adaptive optics on telescopes with large primary mirrors, such as Keck. Current orbital reconstructions are consistent with pure Keplerian motion. However with improved astrometric and radial velocity precision, deviations from pure Keplerian motion are expected. Keck NGAO will be able to detect the deviations from Keplerian motion due to a variety of effects.

Of the theories describing the four fundamental forces of nature, the theory that describes gravity, General Relativity, is the least tested. In particular, General Relativity has not been tested in the strong field limit, on the mass scale of supermassive black holes. The highly eccentric $15 \mathrm{yr}$ orbit of the star S0-2 brings it within 100 AU of the central black hole in the Galactic Center, corresponding to 1000 times the black hole's Schwarzschild radius (event horizon). Studying the pericenter passage of S0-2 and the other high eccentricity stars therefore offers a truly unique opportunity to test General Relativity in the strong gravity regime. 
With Keck NGAO, the orbits can be monitored with sufficient precision to enable a measurement of post-Newtonian General Relativistic effects associated with the black hole. This includes the prograde precession of orbits. As Figure 3 illustrates, the General Relativistic prograde precession can be measured even for single orbits of known stars (e.g., S0-2, $\mathrm{K}=14.1 \mathrm{mag}$ ) if we can achieve an astrometric precision of $\sim 100 \mu$ as coupled with a radial velocity precision of 10 $\mathrm{km} / \mathrm{s}$. These are the key NGAO science requirements derived for this key science driver.

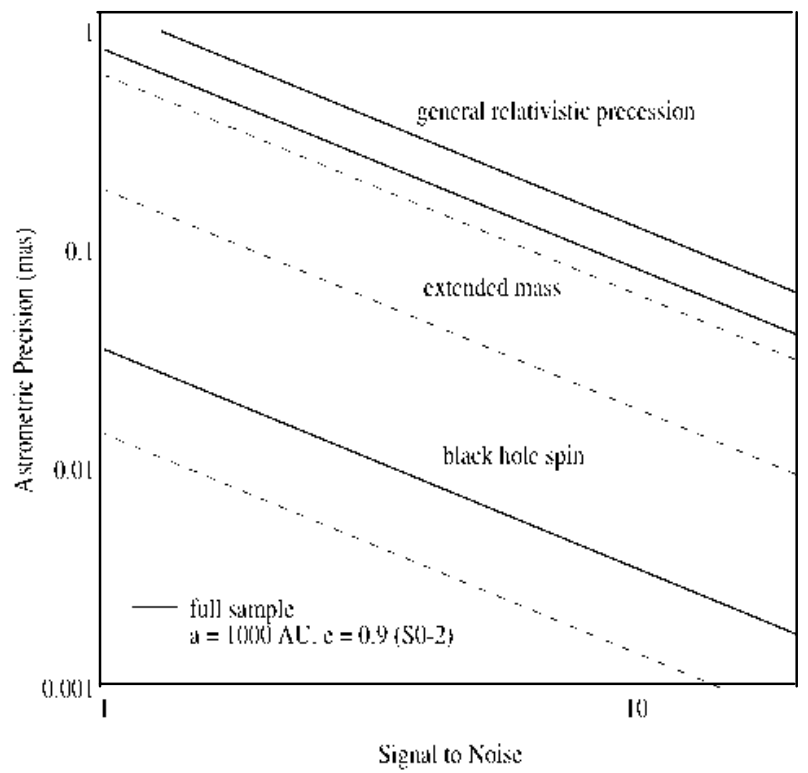

Figure 3. Required astrometric precision for detecting General Relativistic effects at the Galactic Center. From top to bottom, General Relativity effects associated with relativistic prograde precession; extended mass within the stellar orbits; and framedragging effects due to black hole spin (reference $\left[{ }^{12}\right]$ ). Estimates are based on measurements of stellar orbits and positions from Keck diffraction-limited images (thick, solid lines), and assume radial velocity measurement errors of $10 \mathrm{~km} / \mathrm{s}$. The stellar orbits include 16 stars within 0.5 " of Sgr A* with orbital fits obtained from speckle imaging measurements and 142 stars within 1" of Sgr A* with stellar positions obtained with deep AO maps. For comparison, we also show estimates based on measurements from just the short-period star S0-2 (thin, dashed line). Results are for a 10-year baseline with 10 integrations per year. Loworder General Relativity and extended matter effects are detectable at the $>7 \sigma$ level if an astrometric precision of $\sim 100$ $\mu$ as can be achieved. Detection of black hole spin requires either better precision or, at $\sim 100 \mu$ as precision, improved SNR obtained by observation of multiple as-yet-undiscovered higheccentricity, short-period stars over multiple orbits.

Keck NGAO will bring several important improvements to measurements at the Galactic Center:

1) Current measurements are strongly confusion limited, because the Galactic Center is a very crowded field. Higher Strehl at K-band will reduce confusion, improving both photometric and astrometric accuracy because the previously undetected faint star population will cause less of a bias in the positions and magnitudes of brighter stars.

2) Higher Strehl at K-band will allow the detection of new stars, some of which may pass close enough to the black hole to contribute to the obtainable accuracy and precision of General Relativistic effects. (See figure caption.)

3) NGAO's use of multiple laser guide stars and multiple IR tip-tilt stars will decrease the field dependence of the PSF, thereby increasing both photometric and astrometric accuracy.

4) The accuracy of current radial-velocity measurements is limited by signal to noise. NGAO's higher Strehl and lower sky background will materially improve the radial-velocity contribution to orbit determinations.

\subsection{Imaging and Characterization of Extrasolar Planets around Nearby Stars}

The unique combination of the high-contrast near-IR imaging (K-band Strehl ratios $>80 \%$ ) and large sky coverage expected from NGAO will enable direct imaging searches for Jovian-mass planets around nearby young low-mass stars and brown dwarfs. Both the Gemini Observatory and ESO are developing highly specialized planet-finding AO systems with extremely high contrast for direct imaging of young planets. These "extreme AO" systems are very powerful, but their design restricts them to searches around bright, solar-type stars (e.g. I $<9$ mag).

By number, low-mass stars $\left(\mathrm{M} \leq 0.5 \mathrm{M}_{\mathrm{Sun}}\right)$ and brown dwarfs dominate any volume-limited sample, and thus these objects may represent the most common hosts of planetary systems. Such cool, optically faint targets will be unobservable with specialized extreme AO systems because their parent stars are not bright enough to provide a highorder wavefront reference. But thousands of cool stars in the solar neighborhood can be targeted by NGAO because of its laser guide stars. Direct imaging of extrasolar planets is substantially easier around these lower mass primaries, since the required contrast ratios are smaller for a given companion mass. In addition, the very youngest stars in star-forming regions such as Taurus or Ophiucus are generally too faint for extreme AO systems but will be accessible to NGAO. For 
both these science cases, the key angular scales are relatively small (0.1-0.2 arc sec), requiring both the large aperture of the Keck telescopes and careful coronagraph design.

Direct imaging of extrasolar planets by NGAO would measurement of their colors, temperatures, and luminosities, thereby testing theoretical models of planetary evolution and atmospheres. NGAO spectroscopic follow-up will be an important means to characterize the atmospheres of extrasolar planets, which are otherwise inaccessible to spectroscopy (unless their orbits allow transit measurements). Figure 4 summarizes the parameter space explored by NGAO and extreme AO. The complementarity of the two systems is very important: establishing the mass and separation distribution of planets around a wide range of stellar host masses and ages is a key avenue to understanding the planet formation process. The optical faintness of low-mass stars, brown dwarfs and the very youngest stars make them inaccessible to extreme AO systems but excellent targets for NGAO. Required contrast ratios for direct imaging of planets around different classes of low-mass stars and brown dwarfs provide important input to the NGAO science requirements.

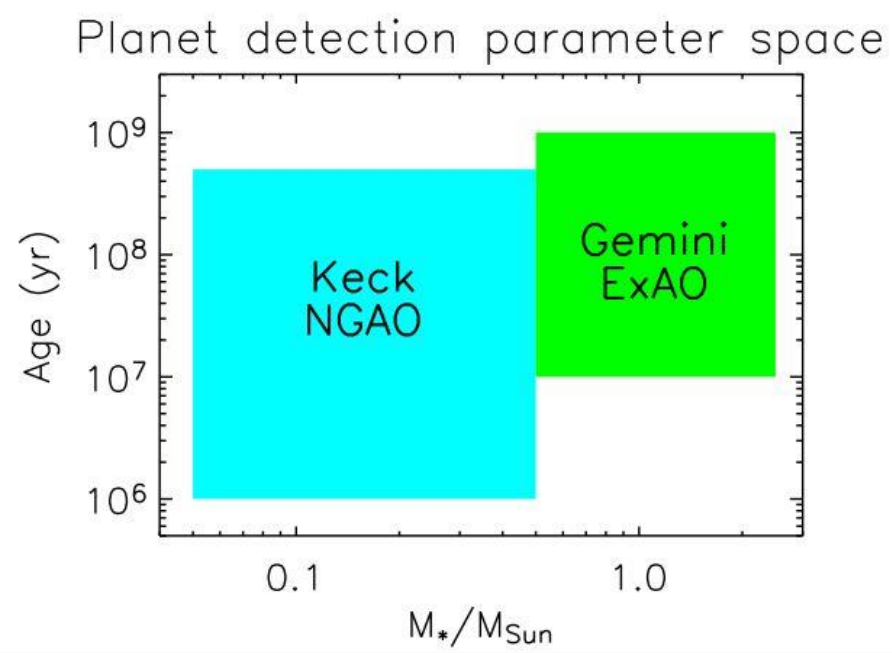

Figure 4. Schematic illustration of the parameter space of Keck NGAO and of the Gemini Planet Imager for direct imaging of extrasolar planets.

The key performance driver for this science case is contrast, not wavefront error. Initial simulations with an RMS wavefront error of $140 \mathrm{~nm}$ indicated that the required contrast could be achieved. Simulations are under way to understand how the science would degrade for wavefront errors of 170 and $200 \mathrm{~nm}$. Excellent control and calibration of internal static wavefront calibration errors and quasi-static errors due to LGS spot shape will also be required of the AO system to minimize quasi-static speckles. Preliminary analytic calculations indicate requirements of 10-30 nm residual static wavefront error and quasi-static LGS-related errors.

\subsection{Multiplicity of Minor Planets in our Solar System}

While space missions largely drove early progress in planetary astronomy, we are now in an era where ground-based telescopes have greatly expanded the study of planets, planetary satellites, and the asteroid and Kuiper belts. Groundbased telescopes can efficiently perform the regular observations needed for monitoring planetary atmospheres and geology, and can quickly respond to transient events.

The study of the remnants from the formation of our solar system provides insight into the proto-planetary conditions that existed at the time of solar system formation. Such information has been locked into the orbits and properties of asteroids and Kuiper Belt objects. The study of binary (and multiple) minor planets is one key path to revealing these insights, specifically by studying their kinematics and geological properties. There are no space missions currently planned to study these binaries.

High angular resolution studies are needed of large samples of binary asteroids to understand how their enormous present-day diversity arose from their formation conditions and subsequent physical evolution, through processes such as collisional disruption and re-accretion, fragmentation, ejecta capture, and fission. Specifically one can study the formation and interiors of minor planets by accurate estimates of the size and shape of minor planets and their companions, and the mass, density, and distribution of interior material by precise determination of the orbital parameters of moonlet satellites. 
For the study of main-belt multiple asteroidal systems, one of the main limitations of current AO observations for a large search for binary asteroids and for characterization of their orbits is the limited number of asteroids observable considering the magnitude limit on today's natural guide star wavefront sensors. The Keck natural guide star AO system can use guide stars down to 13.5 magnitude. With Keck NGAO providing an excellent correction up to tip-tilt star magnitudes $\mathrm{V}=17$ or $\mathrm{J}=19,10 \%$ of the known main-belt population can be searched, corresponding to the potential discovery of 1000 multiple systems assuming the current multiplicity rate of $6 \%-15 \%$.

Closer and fainter satellites should be detectable. To illustrate the gain in quality expected with NGAO, we have generated a set of simulated images of the triple asteroid system 87 Sylvia. The binary nature of this asteroid was discovered in 2001 using the Keck natural guide star AO system. Marchis et al. $\left[{ }^{13}\right]$ announced recently the discovery of a smaller and closer moonlet. The system is composed of a $\mathrm{D}=280 \mathrm{~km}$ ellipsoidal primary around which two moons describe a circular and coplanar orbit: "Romulus", the outermost moonlet $(\mathrm{D}=18 \mathrm{~km})$ at $1356 \mathrm{~km}(\sim 0.7$ ") and "Remus" $(\mathrm{D}=7 \mathrm{~km})$ at $706 \mathrm{~km}(\sim 0.35 ")$. In our simulation we added artificially two additional moonlets around the primary: "S1/New" (D=3.5 km) located between Romulus and Remus (at $1050 \mathrm{~km})$ and "S2/New" $(\mathrm{D}=12 \mathrm{~km})$ closer to the primary (at $480 \mathrm{~km}$ ). This system is particularly difficult to observe since the orbits of the moons are nearly edge-on. We blurred the image using the simulated NGAO and Keck NGS AO PSFs (with an rms error of $140 \mathrm{~nm}$ ) and added Poisson and detector noise to reach a $\mathrm{S} / \mathrm{N}$ of 2000 (corresponding to 1-3 min integration time for a $\mathrm{V}=12$ target). We then estimated whether the moonlets could be detected and their intensity measured by aperture photometry. Figure 5 displays a comparison for one observation between the current Keck NGS AO, NGAO in two wavelengths, and HST/ACS. The angular resolution and thus the sensitivity of the NGAO R-band is a clear improvement and permits detection of the faintest moon of the system.

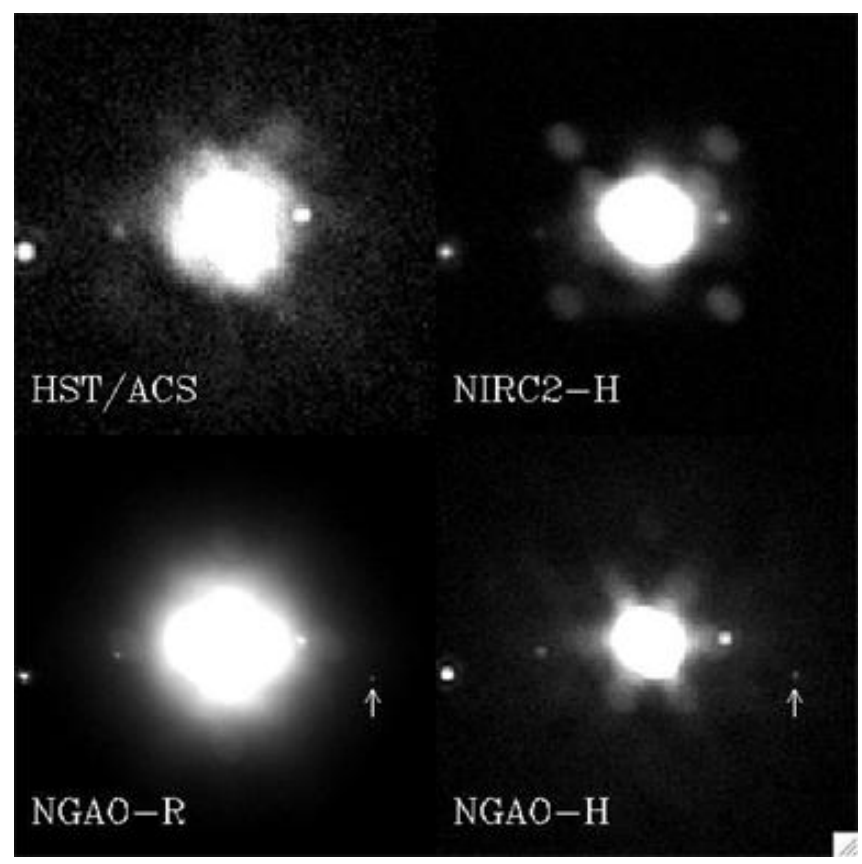

Figure 5. Simulation of pseudo-Sylvia observed with various AO systems and with ACS on the Hubble Space Telescope. We assumed here that NGAO has a wavefront error of $140 \mathrm{~nm}$ in this simulation.

The companion sensitivity (contrast) requirement is $\Delta \mathrm{J} \geq 5.5 \mathrm{mag}$ at 0.5 " separation for a $\mathrm{V} \leq 17$ asteroid $(\mathrm{J} \leq 15.9)$ (asteroid size $<0.2$ ") with a proper motion of $\leq 50$ arcsec/hour. Required observing wavelengths are I through $\mathrm{H}$ bands.

\section{DISCUSSION OF SCIENCE REQUIREMENTS}

We are in the process of flowing down the science requirements derived here to a set of $\mathrm{AO}$ and instrument requirements for the NGAO system in the areas of wavefront error and corresponding sky coverage fractions.

Four of the five Key Science Drivers use very narrow fields:

Black hole mass in nearby AGNs $\quad 5$ arc sec field

General Relativity in the Galactic Center 10 arc sec field

Extrasolar planets around nearby stars $\quad 5$ arc sec field 
Minor planet multiplicity $\quad 3$ arc sec field

The fifth Key Science Driver, Galaxy Assembly and Star Formation History, needs wide fields and high sky coverage. In all Science Cases, infrared tip-tilt stars need to be AO-corrected in order to achieve the required high sky coverage fractions.

Next steps in defining the science requirements for Keck Next Generation AO include refining the requirements for both the Key Science Drivers and the Science Drivers, and iterating with the emerging NGAO system design to maximize the impact of NGAO science.

\section{ACKNOWLEDGEMENTS}

We thank the following participants for their contributions in the process of analyzing the science cases and deriving the science requirements: D. R. Law, C. Steidel, J. Larkin, A. Barth, A. Ghez, J. Lu, B. Macintosh, M. Liu, F. Marchis, T. Treu, L. Melling, P. Marshall, B. Cameron, K. Olsen, J. Melbourne, T. Greene, S. Metchev, L. Hillenbrand, J. Eisner, I. de Pater, and H. Hammel. This work was supported by the following: the W. M. Keck Observatory, and the National Science Foundation Science and Technology Center for Adaptive Optics, managed by the University of California at Santa Cruz under cooperative agreement No. AST-9876783.

\section{REFERENCES}

1 Max, C. E. and E. McGrath, "Keck Next Generation Adaptive Optics Science Case Requirements Document", Keck Adaptive Optics Note 455 (2008).

2 Larkin, J. et al., "OSIRIS: a diffraction limited integral field spectrograph for Keck", Proc. SPIE, 6269, 62691A (2006).

3 Eisenhauer, F. et al., "SINFONI - Integral field spectroscopy at 50 milli-arcsecond resolution with the ESO VLT", Proc. SPIE, 4841, 1548 (2003).

4 Law, D. R. et al., "Predictions and Strategies for Integral-Field Spectroscopy of High-Redshift Galaxies", AJ, 131, 70 (2006).

5 Ferrarese, L. and D. Merritt, "A Fundamental Relation between Supermassive Black Holes and Their Host Galaxies", ApJ, 539, L9 (2000); K. Gebhardt et al., "A Relationship between Nuclear Black Hole Mass and Galaxy Velocity Dispersion", ApJ, 539, L13 (2000).

6 Ghez, A. M. et al., "The First Measurement of Spectral Lines in a Short-Period Star Bound to the Galaxy's Central Black Hole: A Paradox of Youth", ApJL, 586, L127 (2003).

7 Ghez, A. M. et al., "Stellar Orbits around the Galactic Center Black Hole", ApJ, 620, 744 (2005).

8 Schodel, R. et al., "A star in a 15.2-year orbit around the supermassive black hole at the centre of the Milky Way", Nature, 419, 694 (2002).

9 Schodel, R. et al., "Stellar Dynamics in the Central Arcsecond of Our Galaxy", ApJ, 596, 1015 (2003).

10 Shen, Z.-Q. et al., "A size of $\sim 1$ AU for the radio source Sgr A* at the centre of the Milky Way", Nature, 438, 62 (2005).

11 Eisenhauer, F. et al., "A Geometric Determination of the Distance to the Galactic Center", ApJL, 597, L121 (2003).

12 Weinberg, N. N. et al., "Stellar Dynamics at the Galactic Center with an Extremely Large Telescope", ApJ, 622, 878 (2005).

13 Marchis, F. et al., "Discovery of the triple asteroidal system 87 Sylvia", Nature, 436, 822 (2005). 